\title{
Estimación de biomasa y cobertura aérea usando radiometría e imágenes digitales a nivel de campo en pastizales y matorrales
}

\author{
Estimation of biomass and aerial cover using radiometry and field level digital imaging \\ of grasslands and scrublands
}

\author{
Ernesto Chávez Castilloํㅜ Fernando Paz Pellat ${ }^{1 \ddagger}$ y Martín A. Bolaños González ${ }^{1}$
}

${ }^{1}$ GRENASER, Colegio de Postgraduados, Campus Montecillo. Carretera México-Texcoco km 36.5. 56230 Montecillo, Texcoco, Estado de México, México.

${ }^{*}$ Autor responsable (ferpazpel@gmail.com)

\section{RESUMEN}

Para analizar la relación entre las reflectancias de la vegetación obtenidas mediante el uso de sensores ópticos con la cobertura aérea y producción de biomasa de la misma, en el 2007 se realizó una campaña de muestreo en los estados de Jalisco, Zacatecas, Coahuila, Durango, Querétaro y Estado de México, usando radiómetros multiespectrales en condiciones de campo. Adicionalmente a la toma de datos radiométricos se tomaron fotografías digitales para la estimación de la cobertura aérea usando métodos de clasificación supervisada. En aproximadamente el $15 \%$ de los puntos de medición se realizaron muestreos de biomasa aérea. Con el esquema de muestreo utilizado se analizaron las relaciones entre el índice de vegetación (NDVIcp, IVCP y $\beta$ ) con la biomasa y cobertura aérea, estableciendo una relación entre estas variables biofísicas. La estimación de la biomasa y cobertura vegetal de una manera más rápida, como se menciona, permite hacer estimaciones sobre la condición, tendencia o producción de la vegetación natural en pastizales y matorrales, haciendo posible la toma de decisiones sobre el manejo de la misma de manera más eficiente y expedita. El modelo utilizado para obtener la producción de biomasa en pastizal y matorral, a través de datos radiométricos y cobertura vegetal, considerando que los datos procesados corresponden a la fase lineal de crecimiento de la etapa vegetativa, da resultados óptimos para este tipo de vegetación $\left(R^{2}=0.98\right)$.

Cita recomendada:

Chávez Castillo, E., F. Paz Pellat y M. A. Bolaños González. 2017. Estimación de biomasa y cobertura aérea usando radiometría e imágenes digitales a nivel de campo en pastizales y matorrales. Terra Latinoamericana 35: $247-257$.
Palabras clave: cobertura vegetal, índices NDVIcp, $\beta$ e IVCP, imagen digital, datos radiométricos.

\section{SUMMARY}

To analyze the relationship between vegetation reflectances obtained through the use of optical sensors with aerial cover and biomass production, a sampling campaign was carried out in the states of Mexico, Jalisco, Zacatecas, Coahuila, Durango, and Querétaro using multispectral radiometers under field conditions. In addition to radiometric data collection, digital photographs were taken to estimate aerial coverage using supervised classification methods. Approximately $15 \%$ of the measurement points were aerial biomass sampling. The relationship of vegetation index (NDVIcp, IVCP and $\beta$ ) to biomass and aerial cover were analyzed with a sampling scheme, establishing a relationship between these biophysical variables. Estimation of the biomass and vegetation cover in a faster way, allows estimating the condition, trend or production of natural vegetation in grasslands and scrublands, making it possible to make management decisions more efficiently and expedite. The model used to obtain biomass production in grassland and scrubland, through radiometric data and vegetation cover, considering that the processed data correspond to the linear phase of the vegetative growth stage, gives very good results for this type of vegetation $\left(R^{2}=0.98\right)$.

Index words: vegetation cover, IVCP, $\beta$, and NDVIcp, digital imaging, radiometric data. 


\section{INTRODUCCIÓN}

El conocimiento de la distribución intra-anual e interanual de la biomasa forrajera permite la implementación de prácticas de manejo sustentable de los agostaderos, acoplando la dinámica de la oferta de forraje con la demanda alimenticia de los hatos ganaderos (Holechek et al., 1989). En México, la existencia de información de capacidad de carga animal, o su inverso el coeficiente de agostadero, a escala estatal o regional no está actualizada y requiere de aproximarse en forma costo-eficiente para su uso operacional. La COTECOCA (1967) desarrolló estimaciones de coeficientes de agostadero en el periodo 1968-1986, aproximadamente, pero sin una revisión y actualización. Una alternativa para esta tarea es el uso de relaciones clima-producción primaria neta (PPN), las cuales permiten una aproximación general a la capacidad de carga. Así, Díaz-Solís et al. (2003) han usado la relación precipitación con la PPN con resultados satisfactorios. Monterroso et al. (2011) utilizaron la relación evapotranspiración actual con la PPN para parametrizar un modelo de producción forrajera. No obstante la simplicidad de estos enfoques, la disponibilidad de estaciones meteorológicas en zonas de agostadero en México es reducida, independientemente de su actualización, por lo que su uso operacional es restrictivo.

La producción forrajera (porcentaje de la biomasa total disponible) puede estimarse directamente del corte de la biomasa $(\mathrm{Bm})$ o por técnicas indirectas (López et al., 2011); aunque estas últimas no son rápidas y costo-efectivas para escalas de interés regional. Una alternativa operacional es el uso de sensores remotos para realizar estimaciones de la producción forrajera (Tucker y Seller, 1986). Los sensores remotos ópticos en satélites miden la radiación solar reflejada por la superficie terrestre en regiones del espectro electromagnético y la información en las reflectancias puede ser asociada a las características de la vegetación observada, permitiendo su uso en forma espacialmente intensiva y de bajo costo (hoy en día, la mayoría de las imágenes satelitales son sin costo y están fácilmente disponibles). Para este fin se ha usado el alto contraste entre la banda fotosintética del rojo (R) y la no fotosintética del infrarrojo cercano (IRC) para la vegetación (Tucker, 1979). Las bandas del R e IRC han sido usadas para el desarrollo de numerosos índices de la vegetación (IV) (Paz et al., 2007), donde el IV de diferencias normalizadas (Rouse, 1974) $(\mathrm{NDVI}=(\mathrm{IRC}-\mathrm{R}) /(\mathrm{IRC}+\mathrm{R})$, por sus siglas en inglés $)$ es uno de los más populares usados con sensores remotos en plataformas espaciales (Thoma et al., 2002; Medina et al., 2009); aunque su aplicación operacional en términos de percepción de los productores ha sido cuestionada (Rowley et al., 2007).

Un enfoque diferente para estimar la biomasa de la vegetación es a través del uso de la cobertura aérea (COB), en porcentaje, como una variable correlacionada con la biomasa (de la relación del índice de área foliar o IAF con la COB, de acuerdo a la ley de Beer-Lambert: $\mathrm{COB}=100[1-\exp (-\mathrm{kIAF})]$, donde $\mathrm{k}$ es un coeficiente de extinción). Así, la estimación de relaciones entre el NDVI, y otros IV, y la COB se ha utilizado en condiciones de campo con radiómetros espectrales (Post et al., 1999; Calera et al., 2001) y con satélites (Purevdorj et al., 1998), con resultados más o menos satisfactorios.

En este trabajo se presenta una metodología de estimaciones de costos relativamente bajos y rápidas de la biomasa y cobertura aérea de la vegetación, que puede combinarse para aplicaciones regionales, parametrizadas por tipo de vegetación (pastizales y matorrales). La metodología desarrollada fue aplicada en seis estados de la República Mexicana para analizar su viabilidad operacional.

\section{MATERIALES Y MÉTODOS}

\section{Marco Teórico del Uso de Información Espectral e Imágenes Digitales}

El uso de índices espectrales de la vegetación debe basarse en principios teóricos y experimentales sólidos para poder generalizarse. Para entender los principios del diseño de IV, es necesario entender la dinámica del crecimiento de la vegetación asociada al espacio espectral del R-IRC (reflectancias). En la Figura 1 se muestran simulaciones radiativas, mismos patrones que en experimentos (Paz et al., 2007; Huete et al., 1985), de un cultivo o pasto/hierba, donde el crecimiento de la vegetación está definido por las líneas iso-IAF (misma cantidad de vegetación). El cultivo simulado está sobre seis suelos con propiedades ópticas diferentes (pudiendo ser atribuibles a cambios en la humedad, rugosidad, textura, materia orgánica u óxido de hierro), que van de un suelo oscuro (S2) a uno muy claro (S12). Paz et al (2005) detallan estas simulaciones. 


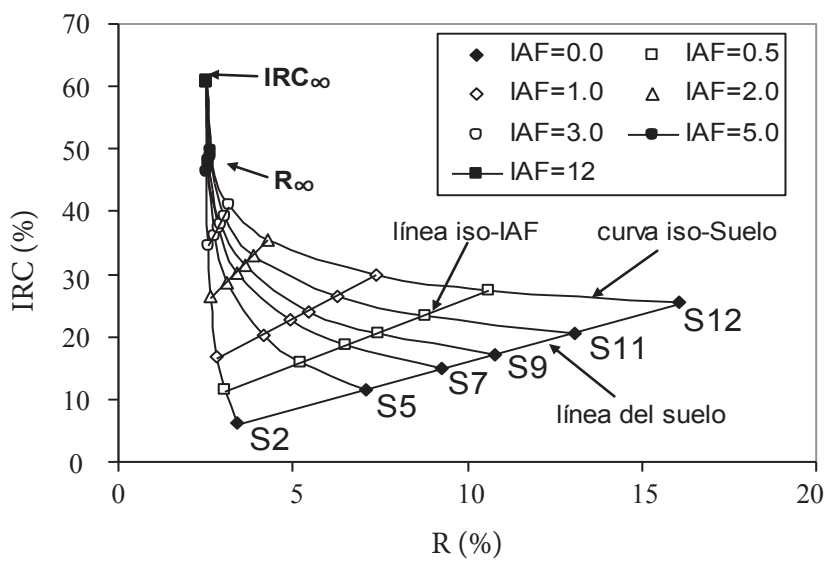

Figura 1. Espacio espectral IRC-R para las simulaciones del cultivo de maíz.

Las líneas iso-IAF (IRC $=\mathrm{a}_{0}+\mathrm{b}_{0} \mathrm{R} ; \mathrm{a}_{0} \mathrm{y} \mathrm{b}_{0}$ dependen del IAF), en la Figura 1, van desde la línea del suelo $\left(\right.$ IRC $=a_{\mathrm{S}}+\mathrm{b}_{\mathrm{S}} \mathrm{R} ; \mathrm{a}_{\mathrm{S}}$ y $\mathrm{b}_{\mathrm{S}}$ son constantes $)$ con IAF $=$ 0 hasta el punto de saturación (las reflectancias no cambian de valor) de las bandas ( $\mathrm{R} \infty$, IRC $\infty$ ), donde el medio es ópticamente denso o de reflectancias en el infinito (Ross, 1981; Huete, 1988). El termino infinito se refiere a que el medio se vuelve infinitamente denso. Para un suelo fijo (propiedades ópticas fijas; es decir, sin cambios), el crecimiento (IAF) sigue líneas iso-suelo, Figura 1.

En la Figura 2a se muestran el patrón entre los parámetros $\mathrm{a}_{0} \mathrm{y}_{0}$ de las líneas iso-IAF, donde hay

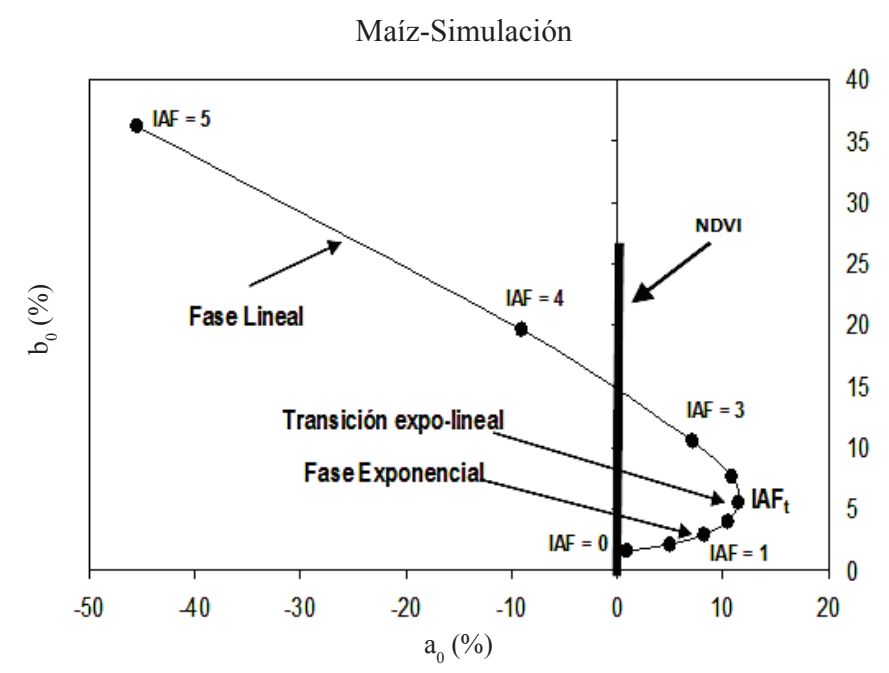

(a) una primera fase tipo exponencial y después un cambio a una lineal (con cambio de pendiente), con una transición entre ellas. La Figura $2 \mathrm{~b}$ muestra los patrones en formato lineal usando la transformación $\mathrm{a}_{0}-1 / \mathrm{b}_{0}$ (Paz et al., 2007).

El caso del NDVI y lo que implica está mostrado en la Figura 2a, donde supone un modelo de una línea recta vertical en el origen, que claramente no aproxima la evidencia teórica y experimental discutida ampliamente en Paz et al. (2005, 2006a y b, 2007, 2014 y 2015), por lo que su uso debe tomarse con cuidado.

Uno de los objetivos primarios del diseño de IV es reducir el efecto del suelo (o estrato debajo de la vegetación) (Romero et al., 2009), de tal manera que los IV midan solo la vegetación, particularmente en el caso de pastizales y matorrales. La mayoría de los IV publicados en la literatura usan una relación lineal entre $\mathrm{a}_{0}$ y $\mathrm{b}_{0}$ para la fase exponencial y la transición exponencial a lineal, Figura $2 \mathrm{a}$, tal como ha sido revisado por Paz et al. (2014 y 2015) para un gran número de IV publicados, incluyendo recientes.

Puesto que para un píxel en una imagen satelital sólo se cuenta con el punto (R, IRC), es necesario saber a qué línea iso-IAF pertenece ( $\mathrm{Paz}$ et al., 2007), problema que implica conocer la reflectancia del suelo. Dado que la cantidad de vegetación en un píxel es independiente de las propiedades ópticas del suelo o de sus cambios, el diseño de los índices de vegetación debe minimizar este efecto. Para hacer esto

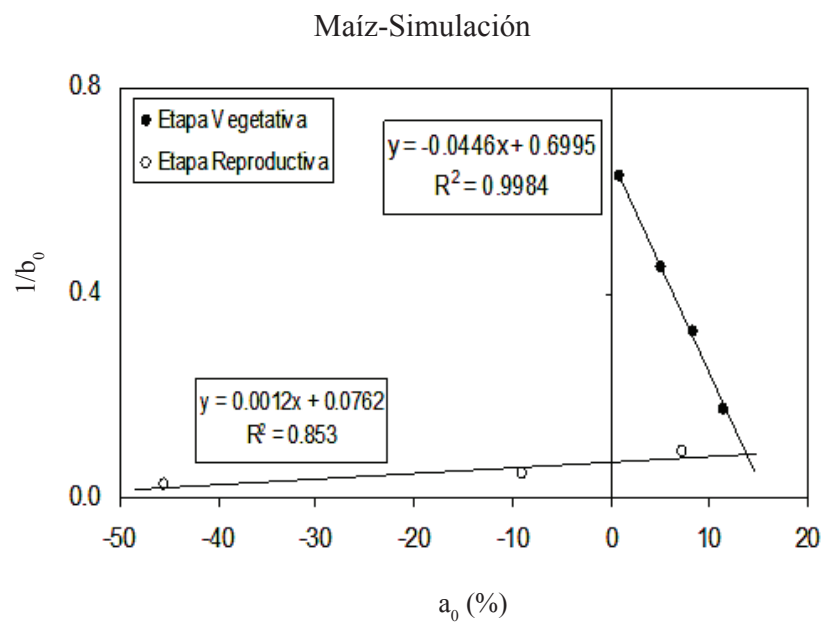

(b)

Figura 2. (a) Patrones $a_{0}-b_{0} y$ (b) $a_{0}-1 / b_{0}$ de las simulaciones radiativas de la Figura 1, discutidas ampliamente en Paz et al. (2007, 2014 y 2015). 
es necesario conocer o, aproximar, la relación entre $\mathrm{a}_{0}$ $\mathrm{y}_{0}$, Figura 2b. En esta perspectiva, Paz et al. (2007) desarrollaron el índice NDVIcp:

$N D V I c p=\frac{\left(I R C-a_{0}\right)-R}{\left(I R C-a_{0}\right)+R}=\frac{b_{0}-1}{b_{0}+1}$

donde: $\mathrm{b}_{0}$ (pendiente de las curvas iso-IAF) se aproxima de la relación (Figura 2b):

$\frac{1}{b_{0}}=c+d a_{0}$

con c y d como constantes empíricas $(\mathrm{c}=1.0 \mathrm{y}$ $\mathrm{d}=-0.0224$, valores de reflectancias en porcentajes).

Substituyendo la relación (2) en la ecuación de las líneas iso-IAF ( $\left.I R C=a_{0}+b_{0} R\right)$ se obtiene una ecuación cuadrática que se resuelve para $\mathrm{b}_{0}$ como ( $\mathrm{Paz}$ et al., 2007):

$b_{0}=\frac{-B+\sqrt{B^{2}-4 A C}}{2 A}$

$A=R$

$B=-\left(\frac{c}{d}+I R C\right)$

$C=\frac{1}{d}$

Un IV alternativo al NDVIcp, que ha mostrado una relación lineal con la cobertura aérea (Romero et al., 2009), es el índice $\beta$ (Paz et al., 2006a):

$\beta=\frac{90-\arctan \left(b_{1}\right)}{45}$

$b_{1}=\frac{b_{0}}{b_{0}-b_{s}}$

$a_{1}=a_{0}\left(1-b_{1}\right)+a_{s} b_{1}$

El índice $\beta$ está basado en el espacio dIRC-IRC, con $\mathrm{dIRC}=\mathrm{IRC}-\left(\mathrm{a}_{\mathrm{S}}+\mathrm{b}_{\mathrm{S}} \mathrm{R}\right)$, transformación lineal del espacio del R-IRC, donde las líneas iso-IAF tienen constantes $a_{1} y_{b_{1}}$. En este espacio, la línea del suelo está sobre el eje IRC y la línea del ápice de la Figura 1 tiene una orientación de $45^{\circ}$. La ventaja de este espacio transformado (dependiente de R e IRC, pero bajo la transformación del dIRC) es que el índice $\beta$ va de 0 (suelo desnudo) a 1 (vegetación densa) y es invariante bajo efectos atmosféricos (Paz et al., 2006b y 2008). Conociendo los parámetros de la línea del suelo (con o sin atmosfera), el NDVIcp también es invariante (da resultados iguales) a efectos atmosféricos (Paz et al., 2006b y 2008). Así, ambos pueden ser usados en aplicaciones con sensores remotos en plataformas espaciales.

El índice NDVIcp tiene una relación lineal con el IAF (Paz et al., 2007), con intercepto nulo cuando se conocen los parámetros de línea del suelo, por lo que suponiendo una relación lineal entre el IAF y la biomasa aérea o Bm (Goudriaan y Van-Laar, 1994), se puede establecer la relación:

$B m=u N D V I c p$

donde: $\mathrm{u}$ es una constante empírica que adquiere valores en función de la vegetación analizada en particular (propiedades ópticas de las hojas, distribución espacial y angular de las hojas, arquitectura del dosel y su distribución geométrica en el área de medición). Esta relación puede usarse bajo condiciones de estrés no demasiado severas, donde la relación se rompe por un cambio de estrategia de la vegetación de almacenar fotosintatos en la parte radical (Stockle et al., 2003).

En las aplicaciones operacionales con satélites de monitoreo de la vegetación de crecimiento anual, considerando que no se conozca los parámetros de la línea del suelo o que exista vegetación perenne, es necesario el seguimiento temporal de la curva espectral del crecimiento para establecer una relación como la (5). Si bien el NDVIcp está relacionado linealmente con la Bm, su patrón temporal es no lineal, pero puede aproximarse usando el inverso del NDVIcp para aproximar un formato lineal, tal como se muestra en la Figura 3 de los datos experimentales de un pastizal natural (Bouteloua spp.) en la cuenca de Walnut Gulch en Arizona, Estados Unidos, cuya descripción detallada se encuentra en Huete et al. (1992), Chehbouni et al. (1994) y Qi et al. (1994).

Ahora bien, considerando que es fácil establecer el inicio del crecimiento anual usando satélites, podemos definir un nuevo índice que aproxime la relación (5), al menos en la etapa vegetativa y de senescencia, como:

$I V C P=\left(\frac{1}{\text { NDVIcp }}\right)_{\min }-\left(\frac{1}{\text { NDVIcp }}\right)$ 


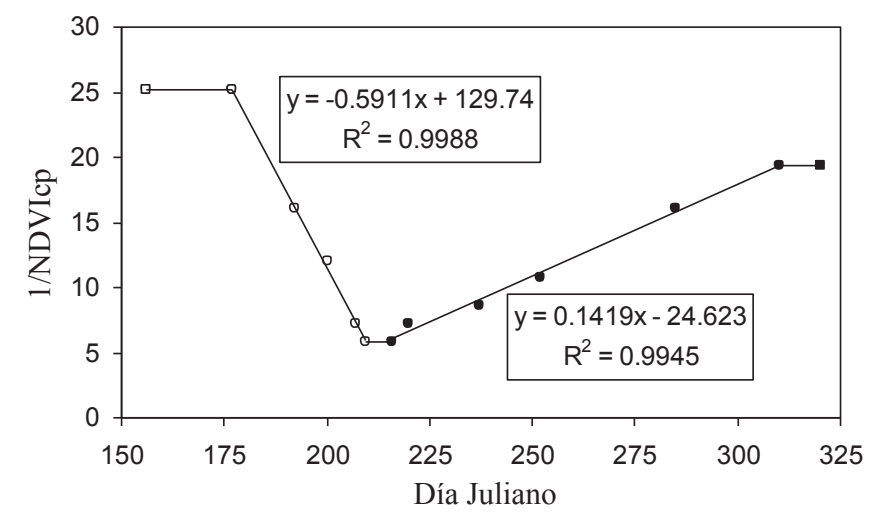

Figura 3. Patrones temporales de 1/NDVIcp para un pasto (Walnut Gulch, Arizona, USA). Los puntos cuadrados fueron puestos en forma artificial para las mesetas.

El valor de (1/NDVIcp) ${ }_{\text {min }}$ se puede obtener de la evolución temporal del índice 1/NDVIcp, Figura 4, y representa el valor al inicio del crecimiento vegetativo, que puede estimarse fácilmente del análisis del patrón temporal de la curva de crecimiento.

En el caso del índice $\beta$, para establecer una relación lineal sin intercepto con la COB, es necesario conocer los parámetros de la línea del suelo, de otra manera existirá una constante aditiva adicional.

\section{Estrategia General de Calibración y Validación en Campo}

Partiendo que la actividad más costosa de muestreo es el corte de la biomasa, el uso de un IV como substituto, permitiría realizar un gran número de muestreos que sean representativos de regiones y de diversos tipos de vegetación y composiciones florísticas. Para esto es necesario calibrar y validar, una relación entre los IV y la Bm usando datos pareados. Para complementar esta actividad, se pueden obtener relaciones entre la cobertura aérea de la vegetación (COB) con un IV, de tal forma que el IV también pueda ser sustituto de la $\mathrm{COB}$ estimada usando fotografías digitales, considerando métodos disponibles en la mayoría de los sistemas de información geográfica (Calera et al., 2001).

De lo anterior, la estrategia general de muestreo planteada es realizar campañas de muestreo en tipos de vegetación homogéneos, donde por alrededor de cada diez mediciones de radiometría y fotografías digitales se realiza una medición de biomasa, radiometría y fotografía aérea, para calibrar las relaciones entre sí. Así, por ejemplo, si se usa un radiómetro para muestreos masivos es posible caracterizar los patrones espaciales de la biomasa usando mediciones indirectas costo-

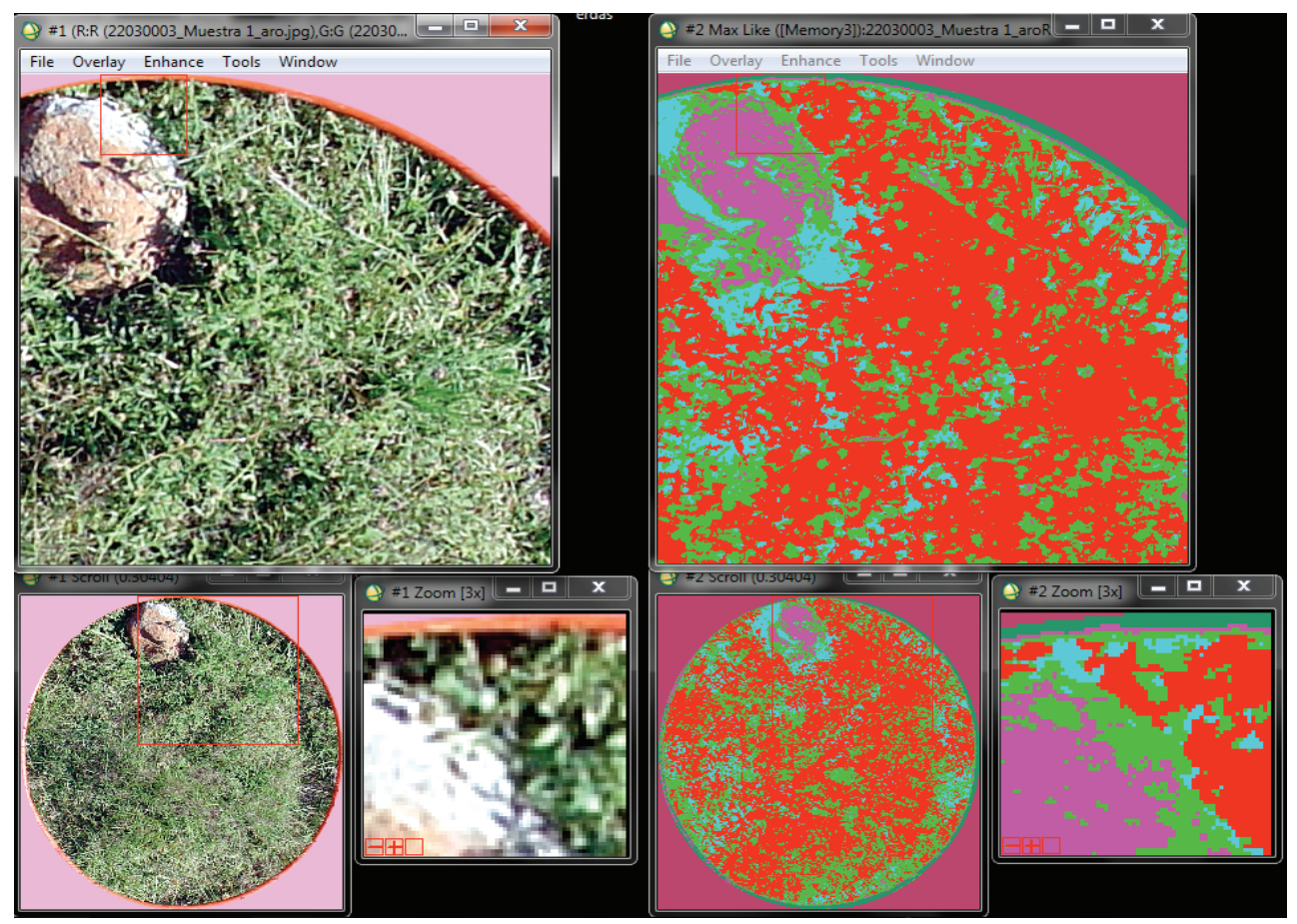

Figura 4. Clasificación supervisada del sitio 03 del estado de Querétaro, referente a la estimación de biomasa. 
efectivas a nivel de campo (radiometría y fotografías digitales), más el proceso en gabinete requerido. La opción clásica es realizar muestreos de biomasa representativos de un tipo de vegetación, requiriendo mayores tiempos para su colecta y pesaje (biomasa fresca y seca).

\section{Muestreos de Campo}

El estudio se realizó en 21 sitios de muestreo que se establecieron en zonas de pastizal y matorral en los estados de Jalisco, Zacatecas, Durango, Coahuila, Querétaro y Estado de México, donde se obtuvo información radiométrica, fotografías digitales (para obtener cobertura aérea) y datos de biomasa.

Para llevar a cabo el muestreo en campo, en cada uno de los sitios de muestreo se trazaron dos líneas de $100 \mathrm{~m}$ de longitud, la primera de Norte a Sur (NS) y la segunda perpendicular a ésta (EO). El total de sitios de muestreo fue de cinco (siete en el Estado de México y Querétaro) por transecto, con el sitio del centro en común para ambos transectos, por lo que el total de muestreos fue nueve (13 en el Estado de México y Querétaro). Los dos extremos de ambas líneas, así como su intersección, se marcaron para próximas visitas de campo, de tal forma que se midiera en los mismos puntos. Las visitas fueron programadas para caracterizar el ciclo del crecimiento anual de la vegetación, desde el inicio de la etapa vegetativa a la senescencia, cuando fue posible. En los estados de Jalisco, Zacatecas, Durango y Coahuila, se llevaron a cabo un total de siete visitas de campo, durante los meses de junio a noviembre de 2007. En el estado de Querétaro, se llevaron a cabo un total de seis visitas de campo durante los meses de septiembre a diciembre de 2007. En tanto que en los sitios del Estado de México se realizaron siete visitas.

En cada sitio se tomaron cuatro fotografías panorámicas, orientadas a cada uno de los puntos cardinales, con la finalidad de captar la evolución del ciclo de crecimiento de la vegetación de cada sitio, a lo largo de las visitas de campo. De la misma forma se tomaron fotografías a nadir en cada punto de medición con la finalidad de estimar el porcentaje de cobertura de la vegetación en cada sitio, mediante técnicas de clasificación de imágenes digitales; simultáneamente a la toma de fotografías a nadir, se realizaron mediciones radiométricas (tres repeticiones en cada punto), utilizando un radiómetro multiespectral Cropscan ${ }^{\mathrm{MR}}$ con 5 bandas similares al sensor TM del satélite Landsat 5. Tanto las fotografías a nadir como las mediciones radiométricas se realizaron a $3 \mathrm{~m}$ sobre el nivel de la superficie (área circular de medición con diámetro de $1.5 \mathrm{~m}$ ), siguiendo las líneas NS y EO.

Adicionalmente, se tomaron mediciones a una altura de $30 \mathrm{~m}$ aproximadamente (área circular de medición con diámetro de $15 \mathrm{~m}$ ), para lo cual se utilizó como plataforma un helicóptero de radio control (Bolaños et al., 2010), desde donde se tomaron fotografías a nadir $\mathrm{y}$, en forma simultánea, se realizaron mediciones radiométricas (tres repeticiones en cada punto), utilizando un radiómetro multiespectral Cropscan ${ }^{\mathrm{MR}}$ de 16 bandas. El objetivo de los vuelos del helicóptero fue analizar el efecto de escala de las mediciones en relación a las mediciones en tierra. Los datos de las mediciones del helicóptero de control remoto no son presentadas en este trabajo, pero tienen patrones similares a los discutidos con las mediciones de campo.

En cada una de las visitas de campo se seleccionaron de dos a cuatro sitios para tomar muestras de biomasa en áreas aledañas a los puntos de medición, que presentaran condiciones de vegetación similares a éstos. En cada uno de estos puntos se tomaron mediciones radiométricas ( 3 repeticiones en cada punto), utilizando el radiómetro multiespectral de 5 bandas a $3 \mathrm{~m}$ de altura y se tomó una fotografía a nadir para el cálculo de la cobertura vegetal ligada a la medición radiométrica. Posteriormente se colocó un arillo de poliducto de 80 $\mathrm{cm}$ de diámetro y se tomó nuevamente otra fotografía a nadir, con la finalidad de estimar el porcentaje de cobertura únicamente dentro del área delimitada por el arillo, que es el área correspondiente a la muestra de biomasa.

Adicionalmente, se midió la altura promedio de la vegetación y se procedió a cortar el total de la misma dentro del arillo de poliducto, para determinar la cantidad de biomasa fresca y seca (después de ser secada a $65^{\circ} \mathrm{C}$ en una estufa por $48 \mathrm{~h}$ ).

A diferencia de los estados de Querétaro y México, en el resto de los estados no se realizaron mediciones pareadas de biomasa y reflectancias, solo de coberturabiomasa y cobertura-reflectancias.

Primeramente se revisó y estandarizó la base de datos radiométricos, se obtuvieron promedios de las tres 
repeticiones tomadas en campo. La geometría solsensor de las mediciones temporales fue estandarizada usando el modelo de Bolaños y Paz (2010), para poder hacer comparables las estimaciones. De cada uno de los sitios de muestreo se concentró un archivo con los datos promediados, también se revisó que no hubiera datos erróneos que pudieran causar algún problema al momento de obtener los valores del índice de vegetación utilizado.

El índice de vegetación utilizado como base fue el NDVIcp, además de los índices de vegetación $\beta$ e IVCP.

\section{Procesamiento de Imágenes Digitales}

La estimación de la cobertura aérea o COB ha sido aproximada usando diferentes técnicas relativamente lentas a nivel de campo (Bolaños et al., 2010), por lo que en nuestro caso se utilizó una clasificación estadística supervisada (Calera et al., 2001) para estandarizar las estimaciones.

El procedimiento consistió de dos fases: la primera en delimitar el área de medición con el radiómetro sobre las fotografías (recorte de imágenes, utilizando el programa Corel Photo-Paint ${ }^{\mathrm{MR}}$ ); y la segunda en determinar la cobertura vegetal a partir de las imágenes recortadas (utilizando el programa $\mathrm{ENVI}^{\mathrm{MR}}$ versión 4.7).

La cobertura aérea se obtuvo a través de un proceso de clasificación supervisada, usando objetos genéricos: vegetación verde, vegetación seca, inflorescencias, suelo, suelo sombreado, piedras y otros (Figura 4), con diferentes tamaños de las superficies de entrenamiento de los objetos (dependientes de la homogeneidad de la distribución espacial en la fotografía). Sólo la cobertura de la vegetación verde se consideró en los análisis, debido a su relación con los IV.

Una vez clasificada la imagen, se determina el número de pixeles, esto para todas las clases encontradas, incluyendo la parte externa al área de interés. El valor de cobertura aérea (\%) se recalcula posteriormente para las clases que se encuentran dentro del área de muestreo del radiómetro, es decir, eliminando la parte correspondiente al "fondo de imagen".

\section{RESULTADOS}

En la Figura 5 se han graficado todos los datos ajustando la relación de cada sitio de muestreo (dividiendo entre la constante $u$ particular para cada tipo de vegetación) para hacer comparables las relaciones, donde el IVCP fue estimado de acuerdo con la relación (5), donde la biomasa $(\mathrm{Bm})$ está dada en $\mathrm{gr} / 0.5 \mathrm{~m}^{2}$, ya que ésta fue el área de muestreo delimitada por un círculo con diámetro de $0.8 \mathrm{~m}$ (área aproximada de $0.5 \mathrm{~m}^{2}$ ).

En esta figura se observan los datos medidos en todos los sitios de muestreo para el Estado de México y Querétaro, después de eliminar los incoherentes (ruidos en el radiómetro). El Cuadro 1 muestra los ajustes del modelo de la relación (5) para cada sitio de muestreo.

Para poder usar de forma equivalente el índice de vegetación $\beta$ o $\mathrm{COB}$, el valor de $\beta$ usado fue estimado del valor de $b_{0}$ a partir de las relaciones definidas

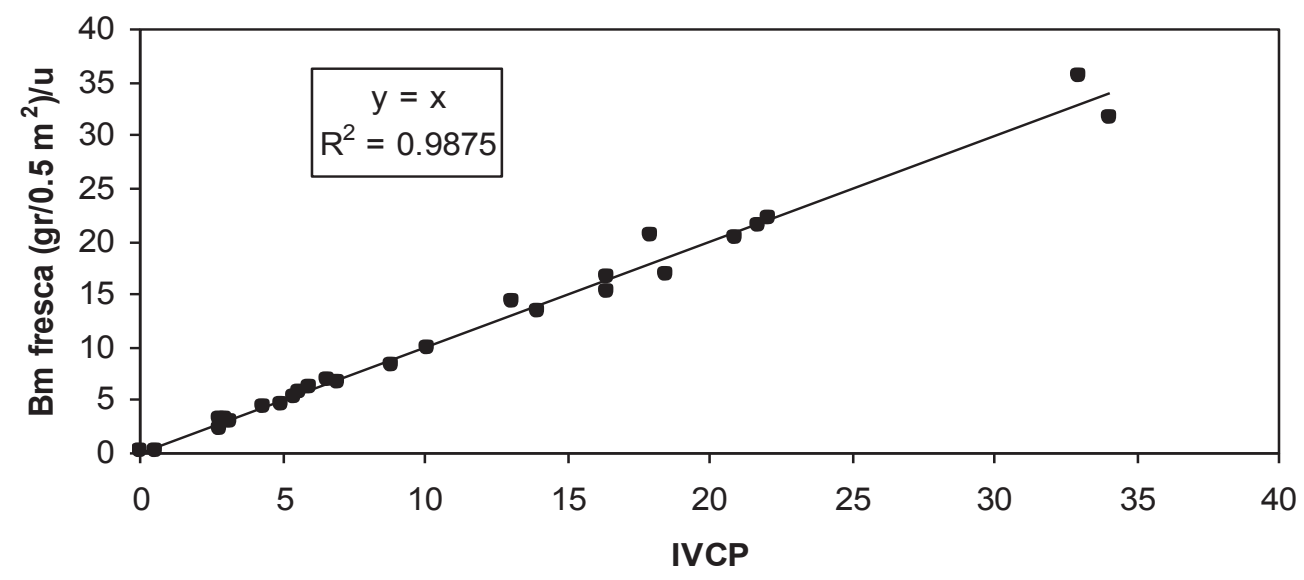

Figura 5. Puntos muestrales del espacio IVCP $(\mathrm{Bm})$ fresca estandarizada para todos los sitios de muestreo del Estado de México y Querétaro. 
Cuadro 1. Resultados del ajuste del modelo lineal entre IVCP-Bm por sitio.

\begin{tabular}{llccc}
\hline Estado & Lugar & Sitio & $\mathrm{u}$ & $\mathrm{R}^{2}$ \\
\hline México & Monjas & 2 & 5.4893 & 0.7120 \\
& Lago de & 1 & 4.8939 & 0.9089 \\
& Texcoco & & & \\
& Colegio de & 3 & 61.440 & 0.9363 \\
& Postgraduados & & & \\
Querétaro & El Sitio & 1 & 18.737 & 0.9179 \\
& & 2 & 45.946 & 0.9865 \\
& Potrero Nuevo & 1 & 32.020 & 0.9909 \\
\hline
\end{tabular}

para el NDVIcp, relaciones (3), y substituido en las relaciones (4) para estimar $\beta$. Esto supone una línea del suelo virtual con parámetros $\mathrm{a}_{\mathrm{S}}=0 \mathrm{y} \mathrm{b}_{\mathrm{S}}=1$ (hipótesis similar usada para la estimación del NDVIcp para las constantes $\mathrm{c}$ y d). En caso de no conocer los parámetros de la línea del suelo, la relación entre COB y $\beta$ estará dada por:

$\beta=s+t C O B$

En la Figura 6 se han graficado todos los datos, ajustando la relación de cada sitio de muestreo (restando s y dividiendo el resultado entre el t particular de cada tipo de vegetación) para hacer comparable la relación COB- $\beta$. En esta figura se muestran los datos muestrales de todos los sitios para el Estado de México y Querétaro, después de eliminar los incoherentes (ruidos en el radiómetro). El Cuadro 2 muestra los ajustes del modelo de la relación (7) para cada sitio de muestreo.

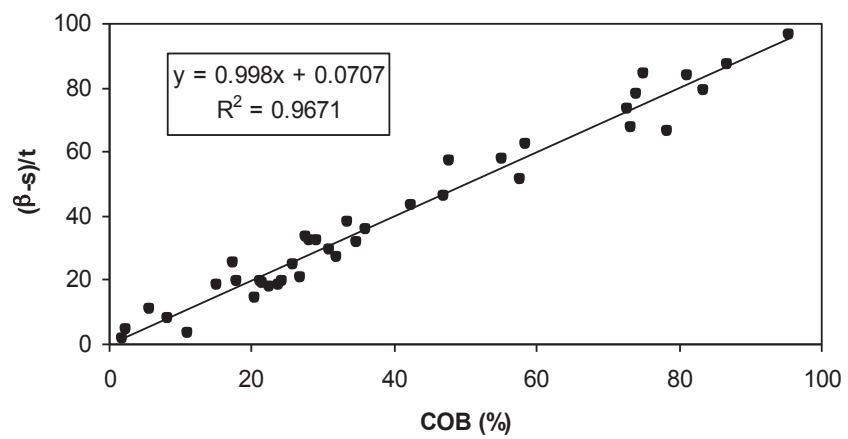

Figura 6. Puntos muestrales del espacio COB- $\beta$ estandarizada para todos los sitios de muestreo del Estado de México y Querétaro.
Cuadro 2. Resultados del ajuste del modelo lineal entre COB- $\beta$ por sitio.

\begin{tabular}{llcccc}
\hline Estado & Lugar & Sitio & $\mathrm{s}$ & $\mathrm{T}$ & $\mathrm{R}^{2}$ \\
\hline México & Monjas & 2 & 0.1185 & 0.0071 & 0.9686 \\
& Lago de & 1 & -0.0449 & 0.0082 & 0.9767 \\
& Texcoco & & & & \\
& Colegio de & 3 & 0.0577 & 0.0023 & 0.5233 \\
& Postgraduados & & & & \\
Querétaro & El Sitio & 1 & 0.0094 & 0.0079 & 0.9570 \\
& & 2 & -0.1291 & 0.0191 & 0.8326 \\
& Potrero Nuevo & 1 & 0.0204 & 0.0124 & 0.8536 \\
\hline
\end{tabular}

A diferencia de los Estados de México y Querétaro, en el resto de los estados no se realizaron mediciones pareadas de biomasa y reflectancias, solo de coberturabiomasa y cobertura-reflectancias; incluyendo los estados de Querétaro y México.

Considerando solo la etapa vegetativa y valores de IAF menores a 3, podemos aproximar la relación entre la $\mathrm{COB}$ y $\mathrm{Bm}$ en forma lineal:

$B m=v+w C O B$

En la Figura 7 se muestra la relación entre COB y $\mathrm{Bm}$ para los datos estandarizados (restando $\mathrm{v}$ y al resultado dividiendo entre $\mathrm{w}$ para cada tipo de vegetación particular) para todos los sitios de muestreo analizados. Los valores ajustados para estimar los parámetros $\mathrm{v}$ y w para cada sitio de muestreo están reportados en el Cuadro 3.

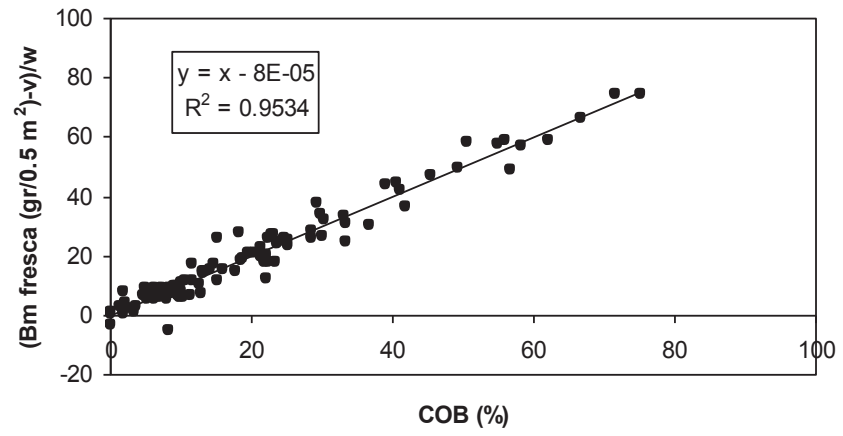

Figura 7. Puntos muestrales del espacio COB-Bm estandarizada para todos los sitios de muestreo. 
Cuadro 3. Parámetros de la relación entre COB-Bm.

\begin{tabular}{|c|c|c|c|c|c|}
\hline Estado & Lugar & Sitio & $\mathrm{v}$ & $\mathrm{w}$ & $\mathrm{R}^{2}$ \\
\hline \multirow[t]{4}{*}{ Coahuila } & Santa Eulalia & 1 & -16.68 & 0.98 & 0.961 \\
\hline & & 2 & -77.82 & 11.57 & 0.957 \\
\hline & & 3 & 49.3 & 6.7 & 0.71 \\
\hline & & 4 & 188.62 & 8.39 & 0.907 \\
\hline \multirow[t]{5}{*}{ Durango } & Rancho Atotonilco & 1 & 27.29 & 4.32 & 0.951 \\
\hline & & 2 & -9.19 & 3.29 & 0.989 \\
\hline & & 3 & -48.61 & 5.07 & 0.972 \\
\hline & & 4 & -13.07 & 1.4 & 0.863 \\
\hline & & 5 & -1.14 & 6.23 & 0.985 \\
\hline \multirow[t]{3}{*}{ Jalisco } & Ojuelos & 1 & -9.66 & 12.43 & 0.787 \\
\hline & & 2 & -18.41 & 9.89 & 0.971 \\
\hline & & 3 & 82.00 & 2.94 & 0.901 \\
\hline \multirow[t]{3}{*}{ Zacatecas } & Juan Aldama & 1 & 4.57 & 5.73 & 0.945 \\
\hline & & 2 & -23.66 & 4.86 & 1.000 \\
\hline & & 3 & 9.47 & 1.22 & 0.965 \\
\hline \multirow[t]{2}{*}{ Querétaro } & El Sitio & 1 & 70.29 & 3.27 & 0.939 \\
\hline & & 2 & 65.73 & 5.31 & 0.955 \\
\hline Querétaro & Potrero Nuevo & 3 & -49.80 & 4.61 & 0.891 \\
\hline \multirow[t]{3}{*}{ México } & Monjas & 1 & 26.86 & 15.7 & 0.882 \\
\hline & Lago de Texcoco & 2 & 50.09 & 2.14 & 0.903 \\
\hline & Colegio de Postgraduados & 3 & 160.63 & 4.37 & 0.875 \\
\hline
\end{tabular}

\section{DISCUSIÓN}

Como se mostró en el apartado de análisis de resultados, las relaciones de los IV con la producción de $\mathrm{Bm}$ y el porcentaje de $\mathrm{COB}$, arrojan para cada modelo específico de cada sitio, en su mayoría un coeficiente de correlación muy alto ( $\mathrm{R}^{2}$ arriba del $90 \%$ ), pero en otros casos se encuentra una correlación baja, como es el caso del sitio 3 del Estado de México en donde la $\mathrm{R}^{2}$ es de alrededor del $50 \%$ y el caso de los sitios 3 de Coahuila y 1 de Jalisco en donde se obtuvieron resultados con $\mathrm{R}^{2}$ alrededor del $70 \%$.

Si consideramos la fecha de toma de los datos en campo y su posterior cálculo en gabinete, los factores o causas que influyeron para obtener correlaciones bajas pueden ser varias: el tipo de vegetación, la condición de manejo, las especies encontradas y su estado vegetativo; así como también en la toma de datos (errores de toma de reflectancias en campo o datos de biomasa), el desplazamiento del punto de toma, confusión de objetos en la clasificación del porcentaje de $\mathrm{COB}$, entre otras.

Para poderestandarizar las relaciones de las variables obtenidas, por medio de regresión lineal se estimaron los valores de los parámetros u, s, t, v y w. Analizando cada situación en particular, las variables relacionadas se ajustan bien, por lo que al estandarizar los modelos obtenidos, se observa que son aplicables para sitios con similares características a los muestreados, ya que al sustituir el valor específico de cada parámetro, el modelo podría aplicarse razonablemente.

Los valores de los parámetros ( $\mathrm{u}, \mathrm{s}, \mathrm{t}, \mathrm{v}$ y w) son dependientes de los tipos de vegetación, por lo que el obtener relaciones generales entre los IV y variables biofísicas es dependiente de las características arquitectónicas, geométricas y ópticas particulares, tal como es esperado de la teoría de transferencia radiativa (Ross, 1981). 


\section{CONCLUSIONES}

- Los resultados indican que al relacionar el IVCP estimado con las mediciones de biomasa y, de la misma forma, el índice $\beta$ con el porcentaje de COB (alcanzando una $\mathrm{R}^{2}=0.98$ y una $\mathrm{R}^{2}=0.96$, respectivamente), se puede utilizar a los IV como un sustituto en la estimación de biomasa o porcentaje de cobertura vegetal para cada condición, ya que los modelos desarrollados son aplicables para este objetivo; de tal forma que los índices de vegetación sirvan para monitorear, evaluar y tomar decisiones en el aprovechamiento y manejo de zonas de pastizal y matorral de una forma más eficiente, sin la necesidad de hacer muestreos destructivos.

- Con el trabajo realizado, en donde se plantea una metodología que se basó en información espectral para estimar la cantidad de biomasa presente en ecosistemas de pastizal y matorral; de acuerdo a los resultados obtenidos, tiene amplias posibilidades de ser aplicada en forma masiva en nuestro país utilizando la información disponible en imágenes de satélite (esencialmente es la misma información que la captada con los radiómetros utilizados, más los efectos atmosféricos), por lo que se tiene la posibilidad de reconstruir la historia de estos recursos (la evolución de los pastizales y matorrales de nuestro país, al menos de los ochentas a la fecha) para que así, en un futuro se puedan actualizar los inventarios en tiempo cortos, sin la necesidad de hacer muestreos de campo, optimizando de esta manera recursos humanos y financieros. El uso de datos radiométricos para calibrar y validar relaciones con la biomasa y cobertura aérea es factible en términos operacionales.

- La aplicación de los desarrollos al caso de sensores remotos en satélites requiere de la consideración de los efectos atmosféricos asociados a las mediciones y el índice NDVIcp (similar para el índice $\beta$ ), que puede eliminarse al conocer los parámetros de las líneas espectrales del suelo (Paz et al., 2006b y 2008) o reducirse de acuerdo a la metodología de Paz et al. (2008).

\section{LITERATURA CITADA}

Bolaños, M. A. y F. Paz. 2010. Modelación general de los efectos de la geometría de iluminación-visión en la reflectancia de pastizales. Rev. Mex. Cienc. Pecu. 1: 349-361.

Bolaños, M. A., F. Paz, E. Palacios y J. Navarro. 2010. Medición de reflectancias en especies forestales utilizando un helicóptero de radio control. Terra Latinoamericana 28: 185-189.

Calera, A., C. Martínez, and J. Melia. 2001. A procedure for obtaining green plant cover: Relation to NDVI in a case study for barley. Inter. J. Remote Sens. 22: 3357-3362.

Chehbouni, A., Y. H. Kerr, J. Qi, A. R. Huete, and S. Sorooshian. 1994. Toward the development of a multidirectional vegetation index. Water Resour. Res. 30: 1281-1286.

COTECOCA (Comisión Técnico Consultiva de Coeficiente de Agostadero). 1967. Metodología para determinar tipos vegetativos, sitios y productividad de sitios. Comisión Técnico Consultiva para la Determinación Regional de los Coeficientes de Agostadero. Publicación No. 8. México, D.F.

Díaz-Solis, H., M. M. Kothmann, W. T. Hamilton, and W. E. Grant. 2003. A simple ecological sustainability simulator (SESS) for stocking rate management on semi-arid grazinglands. Agric. Syst. 76: 655-680.

Goudriaan, J. and H. H. van Laar. 1994 Modelling potential crop growth processes: Textbook with exercises (Current Issues in Production Ecology). Kluwer Academic Publishers. Dordrecht, The Netherlands.

Huete, A. R. 1988. A soil-adjusted vegetation index (SAVI). Remote Sens. Environ. 25: 295-309.

Huete, A. R., G. Hua, J. Qi, A. Chehbouni, and W. J. D. van Leeuwen. 1992. Normalization of multidirectional red and NIR reflectances with the SAVI. Remote Sens. Environ. 41: 143-154.

Huete, A. R., R. D. Jackson, and D. F. Post. 1985. Spectral response of a plant canopy with different soil backgrounds. Remote Sens. Environ. 17: 37-53.

López G., I., J. P. Fontenot y T. B. García P. 2011. Comparaciones entre cuatro métodos de estimación de biomasa en praderas festuca alta. Rev. Mex. Cienc. Pecu. 2: 209-220.

Medina García, G., R. Gutiérrez Luna, F. G. Echavarría Chairz, M. D. Amador Ramírez y J. A. Ruiz Corral. 2009. Estimación de la producción de forraje con imágenes de satélite en los pastizales de Zacatecas. Téc. Pecu. Mex. 47: 135-144.

Monterroso Rivas, A. I., J. D. Gómez, M. L. Toledo, J. A. Tinoco, C. Conde, and C. Gay G. 2011. Simulated dynamics of net primary productivity (NPP) for outdoor livestock feeding coefficients driven by climate change scenarios in México. Atmósfera 24: 69-88.

Paz, F., E. Palacios, E. Mejía, M. Martínez y L. A. Palacios. 2005. Análisis de los espacios espectrales de la reflectividad del follaje de los cultivos. Agrociencia 39: 293-301.

Paz, F., E. Palacios, E. Mejía, M. Martínez y L. A. Palacios. 2006a. Determinación del estado de crecimiento de cultivos usando la transformada de Hough de las reflectividades del follaje. Agrociencia 40: 99-108. 
Paz, F., E. Palacios, L. A. Palacios, L. Tijerina y E. Mejía. 2006b. Correcciones atmosféricas usando patrones invariantes en el espacio del rojo e infrarojo cercano. Rev. Latinoam. Recur. Nat. 2: 3-16.

Paz, F., E. Palacios, M. Bolaños, L. A. Palacios, M. Martínez, E. Mejía y A. Huete. 2007. Diseño de un índice espectral de la vegetación: NDVIcp. Agrociencia 41: 539-554.

Paz, F., M. Bolaños, E. Palacios, L. A. Palacios, M. Martínez y A. Huete. 2008. Optimización del índice espectral de la vegetación NDVIcp. Agrociencia 42: 925-937.

Paz, F., M. E. Romero, E. Palacios, M. Bolaños, J. R. Valdez y A. Aldrete. 2014. Alcances y limitaciones de los índices espectrales de la vegetación: marco teórico. Terra Latinoamericana 32: 177-194.

Paz, F., E. Romero, E. Palacios, M. Bolaños, R. Valdez y A. Aldrete. 2015. Alcances y limitaciones de los índices espectrales de la vegetación: Análisis de índices de banda ancha. Terra Latinoamericana 33: 27-49.

Post, D. F., E. S. Martin, J. R. Simanton, and E. E. Sano. 1999. Use of hand-held radiometers to evaluate the cover and hydrologic characteristics of semiarid rangelands. Arid Soil Res. Rehabilit. 13: 201-217.

Purevdorj T. S., R. Tateishi, T. Ishiyama, and Y. Honda. 1998. Relationships between percent vegetation cover and vegetation indices. Int. J. Remote Sens. 19: 3519-3535.
Qi, J., A. R. Huete, F. Cabot, and A. Chehbouni. 1994. Bidirectional properties and utilization of high-resolution spectra from a semiarid watershed. Water Resour. Res. 30: 1271-1279.

Romero, E., F. Paz, E. Palacios, M. Bolaños, R. Valdez y A. Aldrete. 2009. Diseño de un índice espectral de la vegetación desde una perspectiva conjunta de los patrones exponenciales y lineales del crecimiento. Agrociencia 43: 291-307.

Ross, J. 1981. The radiation regime and architecture of plant stands. Dr. W. Junk. Norwell, MA, USA.

Rouse, J. W., R. H. Haas, J. A. Schell, D. W. Deering, and J. C. Harlan. 1974. Monitoring the vernal advancement of retrogradation of natural vegetation. MASA/GSFC. Type III. Final report. Greenbelt, MD, USA.

Rowley, R. J., K. P. Price, and J. H. Kastens. 2007. Remote sensing and the rancher: Linking perception and remote sensing. Rangeland Ecol. Manage. 60: 359-368.

Stockle, C. O., M. Donatelli, and R. Nelson. 2003. CropSyst, a cropping systems simulation model. Eur. J. Agron. 18: 289-307.

Thoma, D. P., D. W. Bailey, D. S. Long, G. A. Nielsen, M. P. Henry, M. C. Breneman, and C. Montagne. 2002. Short-term monitoring of rangeland forage conditions with AVHRR imagery. J. Range Manage. 55: 383-389.

Tucker, C. J. 1979. Red and photographics infrared linear combination for monitoring vegetation. Remote Sens. Environ. 8: $127-150$.

Tucker, C. J. and P. J. Seller. 1986. Satellite remote sensing of primary production. Inter. J. Remote Sens. 7: 1395-1416. 